\title{
Primary nitrate tolerance in diabetes mellitus
}

\author{
G.McVeigh ${ }^{1}$, G. Brennan ${ }^{2}$, R.Hayes ${ }^{3}$, D. Johnston ${ }^{2}$ \\ ${ }^{1}$ Department of Medicine, University of Minnesota, Minneapolis, Minnesota, USA \\ ${ }^{2}$ Department of Therapeutics and Pharmacology, Belfast City Hospital, Belfast, Northern Ireland \\ ${ }^{3}$ Department of Medicine, Belfast City Hospital, Belfast, Northern Ireland
}

First synthesised in 1846, glyceryl trinitrate was employed clinically as early as 1879 to treat angina pectoris. Today organic nitrates remain a cornerstone in the treatment of chronic stable and unstable angina, acute myocardial infarction, and acute and chronic congestive cardiac failure. The drugs act to produce smooth muscle relaxation that results in dilation of veins, arteries and arterioles. Venodilation that results in pooling of blood in the capacitance vessels occurs at very low plasma concentrations, while arteriolar dilation resulting in a fall in blood pressure and systemic resistance require larger doses of nitrates. The reduction in cardiac preload and afterload decreases myocardial oxygen demand and serves as the main mechanism by which nitrates relieve angina and myocardial ischaemia.

Although organic nitrates have been employed in clinical practice for more than 100 years, their cellular mechanisms of action remain to be fully elucidated. Research in this area has accelerated recently for two main reasons. Firstly, the introduction and widespread use of sustained release oral and transdermal preparations has resurrected the question of nitrate tolerance, which blunts the therapeutic efficacy of the drugs. Secondly, the discovery and identification of nitric oxide, produced via the L-arginine pathway in endothelial cells, has highlighted the importance of nitric oxidecontaining drugs in the control of vascular tone. Exogenous organic nitrates enter the smooth muscle cell and react with thiol groups resulting in denitration and oxidation of the thiols to disulphide. This reaction facilitates the generation of the reactive intermediates nitric oxide and s-nitrosothiols that active guanylate cyclase leading to the production of cyclic guanosine monophosphate. Cyclic guanosine monophosphate in-

Corresponding author: Dr. G. McVeigh, Department of Medicine, Box 741, University of Minnesota, 420 Delaware Street S.E., Minneapolis, MN 55455, USA duces vasodilation by reducing available intracellular cytosolic calcium. Thus exogenous and endogenous nitrovasodilators share the same common pathway to vasodilation through the liberation of nitric oxide.

In a series of experiments we recently found evidence for impaired forearm arteriolar dilator responses to the intra-brachial infusion of acetylcholine (an endothelium-dependent vasodilator) and glyceryl trinitrate (a smooth muscle vasodilator) in patients with Type 2 (non-insulin dependent) diabetes mellitus when compared with non-diabetic control subjects [1]. As evidence exists for endothelial damage in diabetes

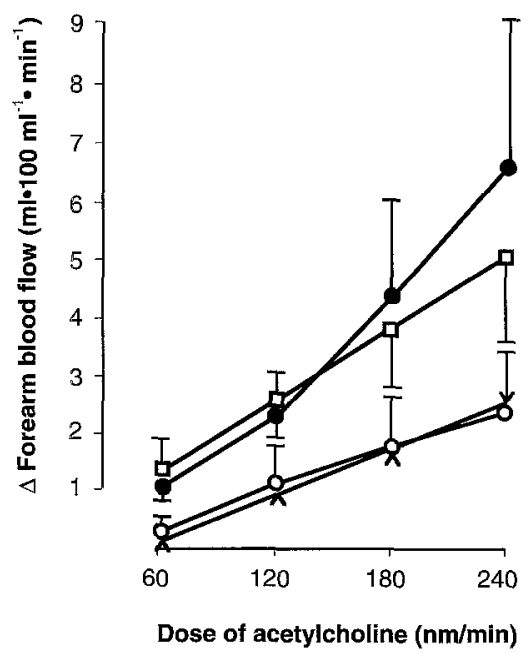

Fig. 1. Change in forearm blood flow (mean change and $95 \%$ confidence intervals shown) to the intra-brachial infusion of acetylcholine in 23 patients with Type 2 diabetes at baseline $0-0$ and after dietary supplementation with olive oil $x-x$ and fish oil $\square-\square$ for 6 weeks and in 21 age- and sex-matched control subjects. Fish oil therapy significantly improved dilator responses to the incremental infusions of acetylcholine when compared with responses recorded at baseline and after ingestion of olive oil in diabetic patients $(p<0.01$ for each dose). Dilator responses recorded after fish oil ingestion in diabetic patients were not different from those reported in healthy control subjects $\bullet$ 


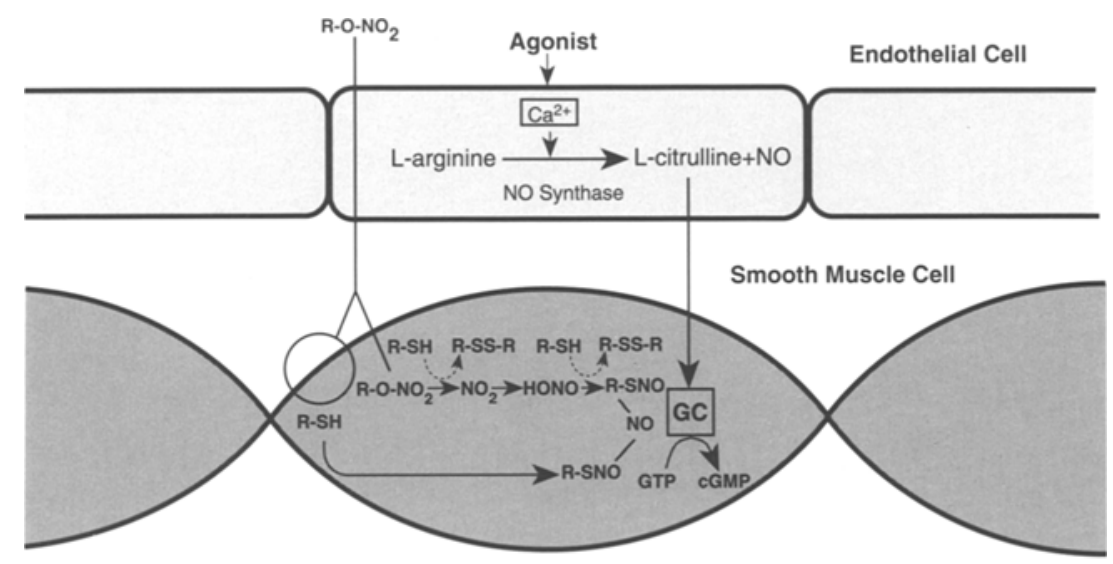

Fig. 2. Schematic depiction of proposed mechanisms by which nitric oxide (NO)-containing substances relax vascular smooth muscle. Organic nitrate esters ( $\mathrm{R}-\mathrm{O}-\mathrm{NO}_{2}$, e.g. glyceryl trinitrate) can react non-enzymatically with thiol-containing groups ( $\mathrm{R}-\mathrm{SH}$ ) which become oxidised to disulphide (R-SS-R). The resulting denitration of organic nitrates ultimately liberates NO that activates the guanylate cyclase (GC) enzyme complex leading to breakdown of guanosine triphosphate (GTP) to cyclic guanosine monophosphate (cGMP). The bioconversion of organic nitrates to $\mathrm{NO}$ may also occur via a membrane bound enzymatic process that requires a thiol-containing compound (most probably glutathione) as a cofactor. NO can also be synthesised from L-arginine in endothelial cells and rapidly diffuses to the underlying smooth muscle to stimulate GC and produce vasorelaxation. The increased oxidative stress associated with diabetes may promote depletion or oxidation of thiol groups thus inhibiting both enzymatic and non-enzymatic production of NO from organic nitrates and limit vascular relaxation. The vasodilator responses to endothelium-derived NO would however remain unimpaired nounced from endothelium-impaired or denuded vessels. Recently Bucala et al. [2] also reported impaired vasodilation to acetylcholine and glyceryl trinitrate in a rat model of experimentally-induced diabetes. They proposed that the deposition of advanced glycation end-products in the subendothelial space quenched nitric oxide produced through the vascular actions of acetylcholine and glyceryl trinitrate. However, the endothelium-dependent vasodilator response to acetylcholine is known to be suppressed within 1 week of sustained hyperglycaemia in experimental diabetes before significant deposition of glycation end-products occurs [3]. Furthermore, as nitric oxide generated from organic nitrates is produced through the interaction with thiol groups in the plasma membrane or within the smooth muscle cell itself, this hypothesis does not appear to fully explain their observations.

Dietary supplementation with omega- 3 polyunsaturated fatty acids improves receptor-mediated vasorelaxation from damaged endothelium to a variety of agonists $[4,5]$. In diabetic patients fish oil ingestion augmented forearm dilator responses to acetylcholine to such an extent that the cumulative dose response curves were no longer different from those recorded in the non-diabetic control group (Fig. 1). By contrast dietary supplementation with olive oil did not alter the vascular responses to acetylcholine. Neither fish oil nor olive oil administration influenced the dilator responses to glyceryl trinitrate. Although a contribution from vasodilator prostaglandins in augmenting receptor mediated dilation with fish oil ingestion could not be excluded, prior studies suggest prostaglandins play a minor role in influencing the vasodilator responses to acetylcholine [6]. In addition, Shimokawa and Vanhoutte [5] found that indomethacin did not alter the augmented endothelium-dependent relaxations to a variety of receptor agonists after dietary administration of codliver oil in porcine coronary arteries. The stereospecific

inhibitor of nitric oxide mediated vasodilation, $\mathrm{N}^{\mathrm{G}}$-monomethyl-L-arginine, attenuated stimulated and possibly basal blood flow responses with fish oil ingestion, further emphasising the role of nitric oxide in influencing the augmented relaxation of the vascular smooth muscle in the diabetic patients [4]. These findings suggest the arteriolar smooth muscle is capable of responding to receptor-operated endothelium-dependent relaxations and excludes structural changes as a common mechanism for the impaired responses to nitrovasodilators, as short-term fish oil therapy would not be expected to alter vessel wall structure.

Biotransformation of organic nitrates requires intracellular sulphydryl groups most probably from cysteine and glutathione to produce the vasoactive intermediates that stimulate guanylate cyclase (Fig. 2). Oxidation or depletion of these sulphydryl donors will impair this metabolic conversion process rendering the vasculature tolerant to organic nitrates. Since the availability of sulphydryl donors is limited, prolonged exposure to nitrate can exhaust this sulphydryl pool with less mobilisation of nitrate and decreased nitric oxide formation. While the development of nitrate tolerance is a multifactorial process this explanation remains the most widely favoured hypothesis to explain the blunted therapeutic efficacy seen with chronic administration of the drugs. In such a state of tolerance however, the vascular response to nitric oxide produced and released from the endothelium is not diminished [7]. In subjects with diabetes anti-oxidant activity reflected by ascorbic acid, alpha-tocopherol and cellular glutathione levels are all decreased $[8,9]$. Thus the increased oxidative stress and heightened free radical activity associa- 
ted with diabetes may alter the reduction-oxidation equilibrium of intracellular thiols and result in primary oxidation or depletion of these essential sulphydryl donors. The magnitude of the impaired dilator responses to the initial administration of glyceryl trinitrate would be dependent on the amount of oxidation or depletion of intracellular thiol groups while the vasculature would remain responsive to nitric oxide generated by the endothelium.

Although some cross-tolerance can occur, vasodilator responses to the administration of the direct acting nitric oxide donor sodium nitroprusside should not be substantially impaired in diabetic patients. Studies in our laboratory indicate that vascular relaxation to sodium nitroprusside is not diminished in diabetic patients compared with control subjects in contrast with responses recorded to the administration of glyceryl trinitrate (unpublished observations). Recent studies have described either no change or a minor impairment in forearm dilator responses to the administration of sodium nitroprusside in Type 1 (insulin-dependent) diabetic patients $[10,11]$.

If intracellular depletion or oxidation of sulphydryl donors results in primary tolerance to the administration of organic nitrates and is of therapeutic importance in diabetes, why has it not been recognised in clinical practice? Firstly, no formal clinical trials investigating the efficacy of nitrates in ischaemic heart disease have been performed exclusively in diabetic patients. Indeed there are many reasons why diabetic patients may be excluded from trials designed to investigate the efficacy of these agents in alleviating the symptoms of ischaemic heart disease. The high incidence of cardiac autonomic neuropathy, peripheral vascular disease and peripheral neuropathy often limits the ability to evaluate diabetic patients with standard treadmill testing. Studies investigating nitrate tolerance in acute ischaemic syndromes utilise a highly selected group of patients with reproducible angina who commonly have a fixed high grade stenosis in one or more coronary arteries at cardiac catheterisation. Virtually without exception diabetic patients appear to have been excluded from these trials. Furthermore, with the widespread recognition that tolerance to nitrates in acute ischaemic syndromes and in heart failure can occur within 24 $48 \mathrm{~h}$ of commencing therapy, a lack of symptomatic and haemodynamic response in diabetic patients may not be regarded with suspicion. For all these reasons it is very possible that important and therapeutically relevant nitrate tolerance may exist as a primary phenome- non in diabetic patients and has been overlooked in clinical practice.

Further studies are required to confirm possible haemodynamic tolerance to the administration of glyceryl trinitrate not only in the arteriolar vasculature but also in the large arteries and venous system. Future studies should also address whether blunted vasodilator responses to glyceryl trinitrate are generic to diabetes in general or whether Type 1 and Type 2 diabetes behave differently. This may be important as some studies suggest that microvascular function, in particular, may differ between the two main types of diabetes.

\section{References}

1. McVeigh GE, Brennan GM, Johnston GD et al. (1992) Impaired endothelium-dependent and independent vasodilation in patients with type 2 (non-insulin-dependent) diabetes mellitus. Diabetologia 35: 771-776

2. Bucala R, Tracey KJ, Cerami A (1991) Advanced glycosylation products quench nitric oxide and mediate defective endothelium-dependent vasodilation in experimental diabetes. J Clin Invest 87: 432-438

3. Lash JM, Bohlen HG (1991) Structural and functional origins of suppressed acetylcholine vasodilation in diabetic rat arterioles. Circ Res 69: 1259-1268

4. McVeigh GE, Brennan GM, Johnston GD et al. (1993) Dietary fish oil augments nitric oxide production or release in patients with type 2 (non-insulin-dependent) diabetes mellitus. Diabetologia 36: 33-38

5. Shimokawa H, Vanhoutte PM (1989) Dietary omega-3 fatty acids and endothelium-dependent relaxations in porcine coronary arteries. Am J Physiol 256: H968-H973

6. Lamontagne D, König A, Bassenge E, Busse R (1992) Prostacyclin and nitric oxide contribute to the vasodilator action of acetylcholine and bradykinin in the intact rabbit coronary bed. J Cardiovasc Pharmacol 20: 652-658

7. Stewart DJ, Holtz J, Bassenge E (1987) Long-term nitroglycerin treatment: effect on direct and endothelium-mediated large coronary artery dilation in conscious dogs. Circulation 75: 847-856

8. Hunt JV, Smith CCT, Wolff SP (1990) Autoxidative glycosylation and possible involvement of peroxides and free radicals in LDL modification by glucose. Diabetes 39: 1420-1424

9. Collier A, Wilson R, Bradley H, Thompson JA, Small M (1990) Free radical activity in Type 2 diabetes. Diabetic Med $727-730$

10. Calver A, Collier J, Vallance P (1992) Inhibition and stimulation of nitric oxide synthesis in the human forearm arterial bed of patients with insulin-dependent diabetes. J Clin Invest 90: 2548-2554

11. Smits P, Kapma J-A, Jacobs M-C, Lutterman J, Thien T (1993) Endothelium-dependent vascular relaxation in patients with Type I diabetes. Diabetes 42: 148-153 